\title{
A propósito do verbo falar no português brasileiro: uma análise em corpus e em bases de dados verbais
}

\author{
Isaac Souza de Miranda Junior ${ }^{1}$, Marcela Monteiro Lemos Couto ${ }^{12}$, Francimeire \\ Leme Coelho $^{1}$, Roana Rodrigues ${ }^{3}$, Oto Vale ${ }^{1}$ \\ ${ }^{1}$ Universidade Federal de São Carlos (UFSCar), São Carlos, Brasil \\ ${ }^{2}$ Instituto Federal de São Paulo (IFSP), Boituva, Brasil \\ ${ }^{3}$ Universidade Federal de Sergipe (UFS), São Cristóvão, Brasil \\ isaacmiranda@estudante.ufscar.br, mmlcouto@gmail.com, \\ flcoelho@estudante.ufscar.br, r.roanarodrigues@gmail.com, \\ otovale@ufscar.br
}

\begin{abstract}
This work investigates the syntactic-semantic proprieties of the verb "falar" (to speak) of Brazilian Portuguese (BP) in a journalistic corpus and three BP verbal databases (VerbNet.Br, VerboWeb and Verbo-Brasil). The data demonstrate a polyvalence and complexity of "falar". In addition, concerning the analysis of the databases, it was verified a need for manual reviews, made out by linguists, and/or expansions of the linguistic descriptions, especially when considering the relevance of constructions with the verb "falar" for different applications in the Natural Language Processing area.
\end{abstract}

Resumo. Neste trabalho, investiga-se o comportamento sintático-semântico do verbo "falar" no português brasileiro $(P B)$ em um corpus jornalístico e em três bases de dados verbais do PB (VerbNet.Br, VerboWeb e Verbo-Brasil). Os dados demonstram a polivalência e complexidade de "falar". Além disso, no que se refere à análise das bases de dados, foi possível constatar a necessidade de revisões manuais, realizadas por linguistas, elou ampliações das descrições linguísticas, principalmente ao considerar a relevância de construções com o verbo "falar" para diferentes aplicações na área de Processamento de Língua Natural.

\section{Introdução}

O verbo é um elemento nuclear das línguas naturais e atua, sobretudo, como responsável pela seleção dos argumentos necessários e essenciais para a construção de uma oração. Há muitas pesquisas descritivas sobre o comportamento dos verbos do português brasileiro, doravante PB, a partir de diferentes abordagens teórico-metodológicas. Podese mencionar trabalhos que se dedicam à descrição morfológica dessa classe de palavras [Bassani 2009], além de investigações de cunho sintático-semântico com propostas de tipologias [Rassi e Vale 2013] e a criação de variadas obras lexicográficas para os usuários comuns da língua [Fernandes 2005 (1940)], [Borba 1990], entre outros.

As descrições formalizadas sobre as construções verbais do PB podem ainda atuar como recursos para diversos empreendimentos na área de Processamento de Língua Natural (PLN), como a sumarização e tradução automáticas e a análise de sentimentos. Em um estudo recente [Rodrigues - no prelo], foram selecionadas, analisadas e 
comparadas três bases de dados verbais do PB (VerbNet.Br, VerboWeb e Verbo-Brasil) ${ }^{1}$, que, ademais de seu inegável valor descritivo em si, contribuem com os recursos disponíveis para o PLN. Em suma, o estudo enfatizou a existência de bases de dados robustas com informações sintático-semânticas sobre os verbos $\mathrm{PB}$ e o papel imprescindível do linguista na elaboração e revisão de dados lexicais para fins computacionais. Além disso, a pesquisa revelou a necessidade de adaptação, ampliação e, até mesmo, a criação de bases de dados verbais que considerem a polivalência e polissemia de determinados verbos e construções para além de seu uso como pleno, incluindo verbos auxiliares, verbos-suporte, ou verbos em expressões multipalavras.

Neste artigo, serão discutidos, mais especificamente, alguns dos aspectos sintático-semânticos do verbo falar no PB. Pode-se mencionar ao menos três motivações principais para a elaboração do presente estudo, a saber: (i) o fato de falar ser um verbo polivalente e bastante frequente na língua; (ii) o valor de falar em construções (diáteses) de comunicação [Couto 2017], que se constituem por um emissor, na posição de sujeito agente, uma mensagem e um destinatário, relevantes para o PLN ao se considerar, principalmente, sua atuação na introdução de discursos reportados que expressam falas (opiniões); e (iii) o comportamento de falar no PB, contrapondo-o ao verbo dizer, demarcando especificidades de usos se comparados a outras línguas naturais, como o espanhol [Humblé 2006] e o inglês [Dehaspe e Eynde 1991]².

Portanto, esta pesquisa se dedica a discutir as seguintes questões: (i) quais os comportamentos sintático-semânticos de falar no PB, a partir de uma análise em corpus; (ii) como o verbo falar está descrito em três bases de dados verbais do PB: VerbNet.Br, VerboWeb e Verbo-Brasil; e (iii) quais informações sobre falar poderiam ser incluídas nas bases analisadas. Sendo assim, espera-se propor uma descrição sintático-semântica de falar, além de divulgar, analisar e contribuir com as bases de dados verbais disponíveis na língua.

Desse modo, este trabalho se organiza da seguinte maneira: na seção 2, são apresentados os usos de falar, encontrados no corpus de notícias do jornal Folha de São Paulo [Santana 2019], com a utilização do software Unitex. Na seção 3, descreve-se e compara-se o lexema falar nas três bases de dados selecionadas nesta investigação. Por fim, na seção 4, são apresentadas as considerações finais e os encaminhamentos para pesquisas futuras.

\section{O verbo falar em corpus}

A fim de verificar como o verbo falar se comporta em uso, além dos conhecimentos introspectivos dos pesquisadores e da classificação proposta por Borba (1990), foram analisadas as suas colocações no corpus do jornal Folha de São Paulo [Santana 2019], que consta de 167.053 notícias. Os dados foram processados pelo Unitex ${ }^{3}$, uma

\footnotetext{
${ }^{1}$ As bases de dados selecionadas no trabalho - VerbNet.Br [Scarton 2013], VerboWeb [Cançado et al., 2018] e Verbo-Brasil [Duran et al. 2013] - sofreram atualizações na última década, estão disponíveis online e de maneira gratuita e apresentam a descrição sintático-semântica de, pelo menos, 1.000 lexemas verbais do $\mathrm{PB}$.

2 As pesquisas citadas destacam especificidades de uso de hablar e decir, em espanhol, e to tell, to say, to speak e to talk, em inglês, contrapondo-as aos casos em que falar atua como sinônimo de dizer, em alguns contextos do PB que introduzem um discurso reportado.

${ }^{3}$ Unitex/GramLab. Disponível em: <https://unitexgramlab.org/pt>. Acesso em: jun. de 2021.
} 
ferramenta plurilingue, de código aberto, que possui dicionários eletrônicos e gramáticas locais para processamento e análise de dados textuais.

Quadro 1. Verbo falar em corpus

\begin{tabular}{|c|c|c|c|c|}
\hline Colocações & Especificidade & Estrutura $^{4}$ & Papéis Temáticos & Exemplo \\
\hline$\varnothing$ & $\begin{array}{l}\text { Construção } \\
\text { intransitiva }\end{array}$ & $\mathrm{N}_{0} \mathrm{~V}$ & $\mathrm{~N}_{0}=$ Agente & $\begin{array}{l}\text { Camila fala } \\
\text { tranquilamente. }\end{array}$ \\
\hline \multirow{6}{*}{ Preposição } & sobre & \multirow{3}{*}{$\mathrm{N}_{0} \mathrm{~V}$ Prep $\mathrm{Nnr}$} & \multirow{3}{*}{$\begin{array}{l}\mathrm{N}_{0}=\text { Agente } \\
\mathrm{Nnr}=\text { Tópico }\end{array}$} & $\begin{array}{l}\text { O deputado não fala } \\
\text { sobre o assunto }\end{array}$ \\
\hline & de & & & $\begin{array}{l}\text { Aquele cara só fala de } \\
\text { economia. }\end{array}$ \\
\hline & em & & & $\begin{array}{l}\text { Dória fala em alterar a } \\
\text { lei. }\end{array}$ \\
\hline & $\operatorname{com}^{5}$ & $\begin{array}{l}\mathrm{N}_{0} \text { V Prep } \\
\text { NHum }\end{array}$ & $\begin{aligned} \mathrm{N}_{0} & =\text { Agente } \\
\text { NHum } & =\text { Co-Agente }\end{aligned}$ & $\begin{array}{l}\text { Machado fala com a } \\
\text { Folha. }\end{array}$ \\
\hline & $\mathrm{a}$ & \multirow{2}{*}{$\begin{array}{l}\mathrm{N}_{0} \text { V Prep } \\
\text { NHum }\end{array}$} & \multirow{2}{*}{$\begin{array}{c}\mathrm{N}_{0}=\text { Agente } \\
\text { NHum = } \\
\text { Destinatário }\end{array}$} & $\begin{array}{l}\text { A candidata falou a uma } \\
\text { multidão. }\end{array}$ \\
\hline & para & & & $\begin{array}{l}\text { Ele fala para uma } \\
\text { plateia de } 300 \\
\text { brasileiros. }\end{array}$ \\
\hline $\begin{array}{l}\text { Complemento } \\
\text { direto }\end{array}$ & $\begin{array}{l}\text { Discurso } \\
\text { reportado }\end{array}$ & $\begin{array}{l}\mathrm{N}_{0} \mathrm{~V} \text { Que } \mathrm{F}_{1} \\
\text { Prep NHum }\end{array}$ & $\begin{array}{c}\mathrm{N}_{0}=\text { Agente } \\
\mathrm{F}_{1}=\text { Mensagem } \\
\text { NHum }_{2}= \\
\text { Destinatário }\end{array}$ & $\begin{array}{l}\text { Falei para os jogadores } \\
\text { que teríamos um desafio } \\
\text { grande. }\end{array}$ \\
\hline $\begin{array}{l}\text { Complemento } \\
\text { direto }\end{array}$ & Nome restrito & $\mathrm{N}_{0} \mathrm{~V} \mathrm{Nr}$ & $\begin{array}{c}\mathrm{N}_{0}=\text { Agente } \\
\mathrm{Nr}=\text { Objeto } \\
\text { [língua }]\end{array}$ & $\begin{array}{l}\text { Ana fala (inglês, } \\
\text { português, árabe) }\end{array}$ \\
\hline $\begin{array}{l}\text { Complemento } \\
\text { direto }\end{array}$ & Nome restrito & $\mathrm{N}_{0} \mathrm{~V} \mathrm{Nr}$ & $\begin{array}{c}\mathrm{N}_{0}=\text { Agente } \\
\mathrm{Nr}=\text { Objeto } \\
\text { [qualidade da } \\
\text { mensagem] }\end{array}$ & $\begin{array}{l}\text { Rui fala (bobagem, } \\
\text { palavrão, besteira, } \\
\text { amenidades, } \\
\text { inverdades). }\end{array}$ \\
\hline $\begin{array}{l}\text { Expressões } \\
\text { multipalavras }\end{array}$ & $\begin{array}{l}\text { Diferentes } \\
\text { estruturas }\end{array}$ & - & - & $\begin{array}{l}\text { Carlos fala (grego, } \\
\text { pelos cotovelos, a } \\
\text { mesma língua que Ana) }\end{array}$ \\
\hline
\end{tabular}

\footnotetext{
${ }^{4}$ Notação da estrutura sintática: $\mathrm{N}_{0}, \mathrm{~N}_{1} \mathrm{~N}_{2}$ : argumentos na posição de sujeito, primeiro complemento e segundo complemento; Nr: argumento preenchido por um nome restrito; Nnr: argumento preenchido por um nome sem restrição; NHum: argumento preenchido por nome humano; $\mathrm{F}_{1}$ : oração; Prep: preposição.

${ }^{5}$ Além da construção com a preposição com, foram encontrados, no corpus, construções pronominais com o verbo falar sempre construídas com sujeito plural. Neste trabalho, entende-se que tais construções são resultado de um processo transformacional de pronominalização, devido à simetria encontrada em: "A [não] fala com B" e "B [não] fala com A", assim "A e B [não] se falam", como no exemplo retirado do corpus: Os irmãos não se falam desde o fim do Oasis.
} 
Ao todo, foram encontradas 49 mil ocorrências com falar $^{6}$ e investigadas 1.470 colocações. No Quadro 1, apresentam-se as construções analisadas, assim como uma proposta de descrição sintática (estrutura) e semântica (papéis temáticos) ${ }^{7}$, seguindo a terminologia adotada pelo modelo teórico-metodológico do Léxico-Gramática [Gross 1975].

Conforme se verifica no Quadro 1, o verbo falar aparece em orações intransitivas, que, por sua vez, geralmente selecionam um circunstancial de modo. Além disso, encontraram-se casos em que falar: (i) seleciona uma preposição, desencadeando diferentes construções sintáticas e semânticas; (ii) seleciona complemento direto e indireto, constituindo um discurso reportado; (iii) seleciona um complemento direto com nome restrito (de idioma/dialeto), que, conforme aponta Borba (1990:740), se refere a uma construção estática, sinonímia de "ter a capacidade de"; (iv) seleciona um complemento com nome restrito, fazendo referência à qualidade da mensagem; e (v) é um constituinte nuclear de expressões multipalavras ${ }^{8}$.

É interessante mencionar que, mesmo em se tratando de um corpus de textos jornalísticos, falar como verbo constituinte de construções comunicativas apresenta caráter informal, sendo encontrado em discursos reportados (marcado por aspas) e/ou em seções com menor formalidade na escrita, como o "caderno de esportes".

\section{3. $O$ verbo falar nas bases de dados verbais do $\mathrm{PB}$}

Conforme mencionado, nesta seção, serão apresentadas as descrições de falar, juntamente com uma análise comparativa com o Quadro 1, na VerbNet.Br, Verbo-Brasil e VerboWeb, bases de dados verbais relevantes do PB, que podem ser utilizadas em diferentes empreendimentos na área de PLN.

A VerbNet.Br [Scarton 2013] é um recurso léxico-computacional (RLC) que agrupa verbos do PB em diferentes classes semânticas. O recurso foi criado de maneira semiautomática a partir do RLC do inglês, a VerbNet [Schuler 2005]. As classes semânticas, inspiradas em Levin (1993), agrupam os verbos de acordo com semelhanças semânticas compartilhadas e suas alternâncias sintáticas.

Por conta da abordagem inter-linguística utilizada na criação desse RLC, as classes presentes na VerbNet.Br são alinhadas diretamente com as classes do inglês. Cada uma delas apresenta as seguintes informações: (i) os membros que a compõem; (ii) os papéis temáticos, que foram herdados da versão do inglês; (iii) as restrições seletivas, que são impostas aos papéis temáticos (como animacidade, concretude etc.), também herdadas do inglês; os frames sintáticos do PB e do inglês, que descrevem a transitividade verbal e, também, itens lexicais selecionados em alternâncias em particular; e (iv) os predicados semânticos, que fornecem informações sobre as relações entre os participantes e o evento da ação verbal.

\footnotetext{
${ }^{6}$ Este número abarcou também as formas nominais a fala e o falar. Neste trabalho, analisaram-se somente as construções verbais.

${ }^{7}$ Os papéis temáticos utilizados na pesquisa baseiam-se no estudo de Santos (2014).

${ }^{8}$ Em Vale (2002) estão descritas e classificadas, sintático-semanticamente, 22 expressões multipalavras com o verbo falar no PB, tais como: falar da boca pra fora ou falar de barriga cheia.
} 
Quadro 2. Verbo falar na VerbNet.Br

\begin{tabular}{|c|c|c|}
\hline \multicolumn{3}{|c|}{ FALAR } \\
\hline Classes & $\begin{array}{c}\text { Papéis temáticos [restrições } \\
\text { seletivas] }\end{array}$ & Alternâncias \\
\hline amuse-31.1 & $\begin{array}{l}\text { Experiencer [+animate] } \\
\text { Stimulus } \\
\text { Result }\end{array}$ & V; V_NP; V_NP_PP [com] \\
\hline chit_chat-37.6 & $\begin{array}{l}\text { Agent [+animate I +organization] } \\
\text { Co-Agent [+animate I +organization] } \\
\text { Topic [+communication] }\end{array}$ & $\begin{array}{l}\text { V; V_PP[com]; V_PP[sobre]; } \\
\text { V_PP[sobre]_PP[com] }\end{array}$ \\
\hline correspond-36.1 & $\begin{array}{l}\text { Agent [+animate I +organization] } \\
\text { Co-Agent [+animate I +organization] } \\
\text { Theme }\end{array}$ & V; V_PP[com]_PP[sobre]; V_PP[sobre] \\
\hline$d u b-29.3$ & $\begin{array}{l}\text { Agent [+animate I +organization] } \\
\text { Theme [+concrete I +organization] } \\
\text { Result }\end{array}$ & V_NP \\
\hline establish-55.5 & $\begin{array}{l}\text { Agent [+animate I +organization] } \\
\text { Theme }\end{array}$ & V_NP \\
\hline lecture-37.11 & $\begin{array}{l}\text { Agent [+animate I +organization] } \\
\text { Topic } \\
\text { Recipient [+animate I +organization] }\end{array}$ & all \\
\hline talk-37.5 & $\begin{array}{l}\text { Agent [+animate I +organization] } \\
\text { Co-Agent [+animate I +organization] } \\
\text { Topic [+communication] }\end{array}$ & $\begin{array}{l}\text { V; V_PP[com]; V_PP[com]_PP[sobre]; } \\
\text { V_PP[para]; V_PP[para]_PP[sobre]; } \\
\text { V_PP[sobre]_PP[com]; } \\
\text { V_PP[sobre]_PP[de]; } \\
\text { V_PP[sobre]_PP[para] }\end{array}$ \\
\hline transfer_mesg-37.1.1 & $\begin{array}{l}\text { Agent [+animate I +organization] } \\
\text { Topic } \\
\text { Recipient [+animate I +organization] } \\
\text { Source }\end{array}$ & $\begin{array}{l}\text { V; V_NP; V_NP_PP[a]; } \\
\text { V_NP_PP[para] }\end{array}$ \\
\hline
\end{tabular}

Nota. Fonte: Adaptado de Scarton (2013).

Conforme se observa no Quadro 2, o verbo falar está presente em oito classes da VerbNet.Br. e muitos frames sintáticos estão presentes em mais de uma classe. É importante salientar que a VerbNet.Br não exemplifica as alternâncias com dados reais do PB ou mesmo criados por introspecção do falante, o que dificulta, em alguns casos, a interpretação dos dados descritos. Para a alternância sintática V_PP[sobre]_PP[de], da classe talk-37.5, por exemplo, não foi localizada nenhuma ocorrência compatível no corpus de dados jornalísticos como se verifica nos dados dispostos no Quadro 1. A pista para o PB é que se trata de um frame com duas preposições (sobrelde) e com a restrição seletiva [+comunication] imposta ao papel temático Topic. Note-se que as preposições sobre e de precedidas do verbo falar selecionam potencialmente argumentos com papel temático assunto (Tópico) ou conteúdo da mensagem (Mensagem), conforme defende Couto (2017). No entanto, no corpus não há ocorrência do verbo falar com essas duas preposições antecedendo o conteúdo da mensagem.

Salienta-se, ainda, o comportamento do verbo falar descrito na classe lecture37.11, o qual possui a descrição all no que diz respeito às alternâncias sintáticas. Isso quer dizer que o verbo falar admite todas as alternâncias sintáticas do PB. Essa é uma 
informação difícil de ser atestada, porque não existe uma delimitação teóricometodológica de quais seriam essas alternâncias ou uma lista das alternâncias do PB para realizar buscas e testes com maior rigor metodológico. Novamente, a ausência de exemplos é um outro grande limitador dessa verificação.

A VerboWeb [Cançado et al. 2018] é uma base digital de consulta lexical, que, devido sua organização e metodologia, pode ser utilizada como RLC. Nela, os verbos estão classificados em função de suas características sintático-semânticas também com base nas classes de Levin (1993). A descrição proposta foi realizada de maneira manual por linguistas e a tipologia verbal ocorre em duas etapas de categorização: classes e subclasses. A classificação em classes consiste em uma divisão dos verbos em função de quatro características: Estrutura sintática básica (disposição sintática dos constituintes: Sintagma Nominal+Verbo, etc.); Papéis temáticos (relação semântica entre um predicado e seus argumentos: agente, paciente, etc.); Decomposição de predicado (demonstração de um predicado em função de predicados primitivos, ou seja, a descrição dos predicados irredutíveis que compõem um predicado complexo: O palestrante falava muito $\Rightarrow[\mathrm{X}$ DO $<$ EVENT>]); Aspecto lexical (classificação dos predicados em função de duração, telicidade e dinamicidade nas classes de Vendler (1967)). Por sua vez, a organização das subclasses consiste no arranjo interno dos verbos de uma classe em função de suas características sintático-semânticas em comum.

\section{Quadro 3. Verbo falar na VerboWeb}

\section{Verbo: Falar}

Exemplo: $O$ palestrante falava muito

Classe: Atividade: verbos internamente causados (inergativos)

Propriedades da Classe:

Conteúdo semântico recorrente na classe: $\mathrm{x}$ faz/produz um evento em si mesmo

- Estrutura sintática básica: [SN V] (verbo intransitivo)

- Estrutura de papéis temáticos: \{Agente\}

- Estrutura de decomposição de predicados: [X DO <EVENT>]

- Aspecto lexical básico: atividade

- Licencia um objeto cognato: $O$ palestrante falou uma fala bonita.

- Licencia um adjunto equivalente ao objeto cognato: $O$ palestrante falou muito bonito.

Subclasse: Verbos de expressão (modo de fala)

Propriedades da Subclasse:

Denota um evento de fala

- Licencia mensagem comunicada na posição de objeto: O palestrante falava muita besteira.

- Licencia a mensagem e o destinatário nas posições de objeto: $O$ palestrante falava muita besteira para o público jovem.

- Licencia um objeto sentencial : O palestrante falava que os jovens são o futuro do país.

- Licencia destinatário na posição de objeto indireto: $O$ palestrante falava animado para o público jovem.

Nota. Fonte: Cançado M., Amaral, L., e Meireles, L. (2021). VerboWeb. Falar.

http://www.letras.ufmg.br/sistemas/verboweb_cliente/ver_verbo.php?id=1220, junho de 2021.

O verbo falar é descrito dentro da VerboWeb como parte da subclasse dos verbos de expressão dentro da classe verbos de atividade internamente causados (inergativos), como é replicado no Quadro 3. 
Com o Quadro 3, pode-se notar que a base descreve dois usos de falar, o primeiro como verbo intransitivo, fazendo parte da classe dos verbos inergativos, e o segundo como verbo de comunicação, referente à subclasse dos verbos de expressão, desconsiderando, no entanto, os casos em que falar ocorre precedido das preposições sobre, de, em e com, presentes no Quadro 1.

Ademais, pode-se reparar também que o "licenciamento de mensagem comunicada na posição de objeto" proposto na VerboWeb faz referência ao mesmo uso (iv) no Quadro 1, porém, o exemplo utilizado ( $O$ palestrante falava muita besteira) não se refere ao conteúdo da mensagem como proposto na VerboWeb, mas sim, como descrito na seção 2, à qualidade da mensagem. Besteira no exemplo, não seria a fala em discurso reportado, mas sim uma qualidade/característica associada a ele pelo locutor.

Por último, a Verbo-Brasil [Duran et al. 2013] é um RLC em que, ao contrário das bases anteriores, os verbos não estão distribuídos em classes maiores de categorização, mas sim descritos individualmente em função dos diferentes sentidos (roleset) que podem assumir. Mesmo que exista dentro da base uma possível classificação (vncls: verbnet class) em função das classes da VerbNet [Schuler 2005], existem sentidos que são vazios quanto essa classificação por não estarem contemplados na VerbNet.

Assim como as outras bases, a Verbo-Brasil é orientada pela descrição das classes de Levin (1993), em que cada verbo é classificado de acordo com suas propriedades sintáticas e aspectos semânticos em comum. Além disso, na Verbo-Brasil, cada sentido é descrito e classificado em função dos seus argumentos e vinculado a um sentido existente no PropBank [Palmer et al. 2005] quando possível. Cada argumento recebe uma identificação de papel temático (vnrole: verbnet role) em função dos papéis temáticos descritos na VerbNet [Schuler 2005].

O verbo falar é descrito dentro da Verbo-Brasil [Duran et al. 2013] com apenas um sentido contendo quatro argumentos que não necessariamente precisam ocorrer juntos em uma sentença. Dentro da descrição de falar, encontra-se também o sentido de duas expressões multipalavras: falar mal e dar o que falar e os seus respectivos argumentos e papéis temáticos. A descrição do sentido de falar pode ser verificada no Quadro 4:

Quadro 4. Verbo falar na Verbo-Brasil

\begin{tabular}{|c|c|c|}
\hline & Sentido & Estrutura Argumental \\
\hline Falar & $\begin{array}{l}\text { Roleset id: falar. } 01 \text {, dizer; } \\
\text { declarar; vncls: } 37.7 \text {; } \\
\text { Mapeamento para o inglês: } \\
\text { say. } 01 \text {, talk. } 01 \text {, speak. } 01\end{array}$ & $\begin{array}{l}\text { Arg0: falante (vnrole: } 37.7 \text {-agent) } \\
\text { Arg1: idioma falado; assunto (falar de, falar sobre, } \\
\text { falar contra, falar a favor de) ou elocução (vnrole: } \\
\text { 37.7-topic) } \\
\text { Arg2: ouvinte, interlocutor (falar a, falar para, } \\
\text { falar com) (vnrole: } 37.7 \text {-recipient) } \\
\text { Arg3: atributo do arg0 (falar como) }\end{array}$ \\
\hline
\end{tabular}

Nota. Fonte: Duran, M. S., Martins J. P., Coimbra, M., Patire, P. A., Hartmann N., e Aluísio, S. (2014). Verbo-Brasil. Framefile-falar-v. http://143.107.183.175:21380/verbobrasil/textoFrames/falar-v.html, junho de 2021.

\footnotetext{
${ }^{9}$ A construção falar como foi encontrada no corpus mas, por não se tratar de um argumento selecionado pelo verbo, não foi inserida no Quadro 1 .
} 
A forma como falar está descrito no Quadro 4, mesmo que abrangente, pode gerar alguns problemas de descrição, uma vez que o roleset falar.01 apresenta 32 exemplos $^{10}$ diferentes de construções com o verbo, em que cada construção tem especificidades sintáticas distintas, apresentando números distintos de argumentos. Vale ressaltar também que em alguns exemplos da Verbo-Brasil, como o roleset falar.01 exemplo 2 (Não ouvi falar nada sobre arbitragens) e o roleset falar.01 exemplo 30 (Takuo Hirano e Tetsuyuki Hirano, pai e filho, vieram falar sobre o novo conceito de design que estão desenvolvendo), são representados na base com estruturas argumentais idênticas (contando apenas com arg1), o que é um problema considerando que ambos os exemplos apresentam estruturas distintas, enquanto que no exemplo 2 não há a presença explícita de um arg0, no exemplo 30 Takuo Hirano e Tetsuyuki Hirano é arg0 não somente de vir, mas como também de falar.

Como visto na seção, cada base exprime uma cobertura distinta dos usos de falar, isso ocorre devido à polivalência e polissemia de falar, reforçando a necessidade da revisão e ampliação das descrições disponíveis sobre esse verbo nas bases de dados analisadas.

\section{Considerações finais}

Por se tratar de um verbo bastante frequente, o comportamento do verbo falar apresenta um número considerável de empregos que merecem ser considerados em qualquer trabalho descritivo. Esse é um fato comum em itens de alta frequência que apresentam, via de regra, uma polissemia que nem sempre é detectável à primeira vista.

De fato, retomando as questões que nortearam esta pesquisa, nota-se que o verbo falar no PB apresenta um comportamento sintático-semântico variado, que requer estudos aprofundados. Além dos casos recenseados no Quadro 1, destaca-se ainda a necessidade de análises dedicadas à polivalência e polissemia de falar em construções como: falar em ( seu discurso + casamento + inglês...), falar (como + na condição de + na qualidade de) (presidente + professor) e falar (por + no lugar de) alguém. Tais complementos poderiam ser atribuídos à estrutura argumental do verbo? Esse tipo de pergunta poderia ser estendida a outras construções de comunicação, considerando-se o comportamento de diferentes verba dicendi [Costa e Freitas 2017] [Baptista 2010].

No que se refere ao estudo de falar nas bases de dados verbais, verificou-se que todas as bases enfatizam o uso de falar em construções de comunicação. Constatou-se ainda que as bases verbais construídas para fins computacionais (VerbNet.Br e VerboBrasil) necessitam de uma revisão linguística especializada, seja para incluir exemplos reais da língua, seja para refinar a descrição e propor classificações mais granulares dos dados. Por sua vez, a base que se constrói sob descrições linguísticas manuais (VerboWeb) parece ser a que possui maior coerência teórico-metodológica, embora apresente um número reduzido de construções analisadas, podendo ampliar o estudo de tal verbo em futuras atualizações da base.

Sendo assim, acredita-se que as informações sobre o verbo falar levantadas no corpus e descritas neste trabalho podem impulsionar não só ao incremento e atualização das bases de dados verbais elencadas, como também à realização de novos estudos sintático-semânticos sobre esse verbo.

\footnotetext{
${ }^{10}$ Os exemplos da Verbo-Brasil foram extraídos do corpus PLN-Br [Bruckschen et al. 2008].
} 


\section{Agradecimentos}

Os autores agradecem à CAPES (Código Financeiro 001) e ao Centro de Inteligência Artificial (C4AI) da Universidade de São Paulo, apoiado pela IBM e FAPESP $\left(\mathrm{n}^{\circ}\right.$ 2019/07665-4).

\section{Referências}

Baptista, J. (2010). Verba dicendi: a structure looking for verbs. In: Nakamura, T., Laporte, E., Dister, A., Fairon, C. Les Tables - La grammaire du français par le menu. Mélanges en hommage à Christian Leclère. Cahiers du CENTAL, 6, 11-20.

Bassani, I. S. (2009). Formação e interpretação dos verbos denominais do português do Brasil. Dissertação (Mestrado em Semiótica e Linguística). São Paulo, Universidade de São Paulo.

Borba, F. S. (coord.). (1990). Dicionário gramatical de verbos do português contemporâneo do Brasil. São Paulo: Editora UNESP.

Bruckschen, M., Muniz, F., Souza, J. G. C., Fuchs, J. T., Infante, K., Muniz, M. e Aluísio, S. M. (2008). Anotação linguística em XML do corpus PLN-BR. Série de relatórios do NILC, NILC-ICMC-USP.

Cançado, M., Amaral, L., e Meirelles, L. L. (2018). Verboweb: Uma proposta de classificação verbal. Revista da Anpoll, 1(46), 123-141.

Cançado M., Amaral, L., e Meireles, L. (2021). VerboWeb. Falar. http://www.letras.ufmg.br/sistemas/verboweb_cliente/ver_verbo.php?id=1220, Junho de 2021.

Costa, B. F. S., e Freitas, C. (2017). Verbos de elocução em português: um estudo descritivo com base em grandes corpora e motivado pela linguística computacional. Fórum Linguístico, 14(3), 2266-2285.

Couto, M. M. L. (2017). O estudo das valências verbais aplicado às construções de comunicação do português brasileiro. Dissertação (Mestrado em Estudos Linguísticos). Belo Horizonte, Universidade Federal de Minas Gerais.

Dehaspe, L. e van den Eynde, K. (2012). The Pronominal Approach to Verbal Valency: A formal description of speak, say, tell, and talk. In Betriebslinguistik und Linguistikbetrieb (pp. 273-280). Max Niemeyer Verlag.

Duran, M. S., Martins, J. P., e Aluisio, S. M. (2013). Um repositório de verbos para a anotação de papéis semânticos disponível na web. In: Proceedings of the 9th Brazilian Symposium in Information and Human Language Technology. <https://www.aclweb.org/anthology/W13-4820.pdf>, acesso em junho de 2021.

Duran, M. S, Martins J. P., Coimbra, M., Patire, P. A., Hartmann N. e Aluísio, S. (2014). Verbo-Brasil.

Framefile-falar-v. http://143.107.183.175:21380/verbobrasil/textoFrames/falar-v.html>, acesso em junho de 2021.

Fernandes, F. (2005 [1940]). Dicionário de verbos e regimes. 45 ed. Porto Alegre: Globo. 
Gross, M. (1975). Méthodes en Syntaxe: régime des constructions complétives. Paris: Hermann,

Humblé, P. (2006). Falsos cognados. Falsos problemas. Un aspecto de la enseñanza del español en Brasil, Revista de Lexicografía, 2005-2006, 12: 197-207 <https://ruc.udc.es/dspace/handle/2183/5510>, acesso em junho de 2021.

Levin, B. (1993). English Verb Classes And Alternations: A Preliminary Investigation. Chicago: University of Chicago Press.

Palmer, M., Gildea, D., e Kingsbury, P. (2005). The proposition bank: An annotated corpus of semantic roles. Computational linguistics, 31(1), 71-106.

Rassi, A. P. e Vale, O. A. (2013). Tipologia das construções verbais em PB: uma proposta de classificação do verbo dar. Caligrama, Belo Horizonte, v. 18, n. 2.

Rodrigues, R., Lemos-Couto, M. M., Coelho, F. L., Miranda Jr, I. e Vale, O. (2021) Bases de dados verbais do português brasileiro (artigo em vias submissão).

Santana, M. (2019). "News of the Brazilian Newspaper - 167.053 news of the site Folha de São Paulo (Brazilian Newspaper)", <https://www.kaggle.com/marlesson/news-ofthe-site- -folhauol>, acesso em junho de 2021.

Santos, R. P. T. (2014). Automatic Semantic Role Labeling for European Portuguese. Dissertação (Mestrado em Ciências da Linguagem). Universidade do Algarve, Faro.

Scarton, C.E. (2013). VerbNet.Br: construção semiautomática de um léxico verbal online $e$ independente de domínio para o português do Brasil. Dissertação (Mestrado em Ciências de Computação e Matemática Computacional). Universidade de São Paulo, São Carlos.

Schuler, K. K. (2005). VerbNet: A broad-coverage, comprehensive verb lexicon. University of Pennsylvania.

Vale, O. A. (2002). Expressões cristalizadas do português do Brasil: uma proposta de tipologia. Tese (Doutorado em Linguística e Língua Portuguesa). Universidade Estadual Paulista Júlio de Mesquita Filho, Araraquara.

Vendler, Z. (1967). Linguistics in Philosophy. Ithaca, NY: Cornell Univ. Press. 